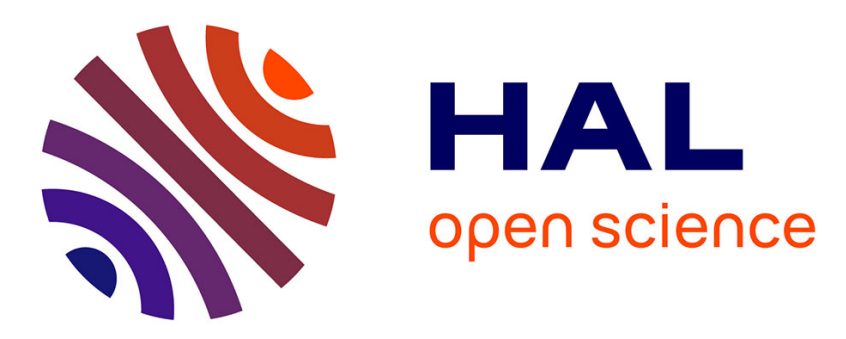

\title{
Territorialiser l'environnement, un objectif pour la géographie
}

\author{
Claude Bertrand, Georges Bertrand
}

\section{To cite this version:}

Claude Bertrand, Georges Bertrand. Territorialiser l'environnement, un objectif pour la géographie. Géographes associés, 1992, Sens et pratiques de l'environnement. Spécial Géoforum 91, 10, pp.63-74. 10.3406/geoas.1992.1768. hal-02569823

\section{HAL Id: hal-02569823 \\ https://hal.science/hal-02569823}

Submitted on 11 May 2020

HAL is a multi-disciplinary open access archive for the deposit and dissemination of scientific research documents, whether they are published or not. The documents may come from teaching and research institutions in France or abroad, or from public or private research centers.
L'archive ouverte pluridisciplinaire HAL, est destinée au dépôt et à la diffusion de documents scientifiques de niveau recherche, publiés ou non, émanant des établissements d'enseignement et de recherche français ou étrangers, des laboratoires publics ou privés. 


\section{Territorialiser l'environnement, un objectif pour la géographie}

\section{Claude Bertrand, Georges Bertrand}

\section{Résumé}

De périphérique, l'environnement s'est glissé au coeur du social d'où il interroge et Bouscule l'ensemble des sciences humaines. La géographie, science traditionnelle des relations des hommes avec leurs milieux, est aujourd'hui en mal d'environnement. La géographie ne doit pas seulement se situer par rapport à l'écologie. Elle doit partir d'une démarche spécifique : territorialiser l'environnement ; c'est-à-dire l'enraciner dans l'espace naturel et social, l'anthropiser, l'hybrider, l'historiser, le patrimonialiser... Cela suppose que la géographie redéfinisse certains de ses objectifs et de ses méthodes, en particulier, réintègre la nature et les phénomènes spatiaux qui en dérivent au centre de ses réflexions et de ses pratiques.

\section{Citer ce document / Cite this document :}

Bertrand Claude, Bertrand Georges. Territorialiser l'environnement, un objectif pour la géographie. In: Géographes associés n¹0, 1er semestre 1992. Sens et pratiques de l'environnement. Spécial Géoforum 91 . pp. 63-74;

doi : https://doi.org/10.3406/geoas.1992.1768

https://www.persee.fr/doc/geoas_1266-4618_1992_num_10_1_1768

Fichier pdf généré le 09/11/2019 


\section{TERRITORIALISER}

L'ENVIRONNEMENT

UN OBJECTIF POUR

LA GÉOGRAPHIE

\section{CLAUDE ET GEORGES BERTRAND}

CIMA-URA 366du CNRS,

Institut de Géographie Daniel Faucher, UNIVERSITÉ DE TOULOUSE-LE-MIRAIL

"Car maintenant la terre nous tient"

Michel Serres - Le Contrat naturel

"L'environnement bouscule les sciences"

Jean-claude Oppeneau - SRETIE Ministère de l'Environnement

RÉSUMÉ : De périphérique, l'environnement s'est glissé au coeur du social d'où il interroge et bouscule l'ensemble des sciences humaines. La géographie, science traditionnelle des relations des hommes avec leurs milieux, est aujourd'hui en mal d'environnement. La géographie ne doit pas seulement se situer par rapport à l'écologie. Elle doit partir d'une démarche spécifique : territorialiser l'environnement ; c'est-à-dire l'enraciner dans l'espace naturel et social, l'anthropiser, l'hybrider, l'historiser, le patrimonialiser... Cela suppose que la géographie redéfinisse certains de ses objectifs et de ses méthodes, en particulier, réintègre la nature et les phénomènes spatiaux qui en dérivent au centre de ses réflexions et de ses pratiques.

MOTS CLÉS : Environnement - Aménagement - Géographie - Histoire - Ecologie Territoire - Nature - Epistémologie - Méthode

\section{LA GÉOGRAPHIE FRANÇAISE EN MAL D'ENVIRONNEMENT}

L'expression "géographie de l'environnement" a toujours sonné faux aux oreilles des géographes : tautologie pour les uns, fourvoiement pour les autres. La géographie française ne semble pas s'être remise d'avoir été, au cours du XIX" siècle, la "science du milieu", préfiguration heureuse des études sur l'environnement, et d'avoir épuisé cette veine par incapacité à se renouveler aussi bien au plan de l'objet d'étude qu'à celui de la méthode. Aujourd'hui, la relation entre les recherches géographiques et les études environnementales ne va pas de soi. Elle est pourtant inévitable. L'avenir de la géographie passe par l'adhésion critique et constructive aux problématiques et aux thématiques de l'environnement.

C'est d'ailleurs avec une prudence certaine que le sujet est abordé à Géoforum 1991 par le biais du "Sens et pratique de l'environnement : du côté des géographes". 
"A côté des géographes" aurait été provocant, mais certainement plus juste tant les recherches fondatrices de l'environnement se sont élaborées sur des bases écologiques, hors du champ de la géographie. Déploiement foudroyant qui, en moins de deux décennies, a bouleversé, non sans excès et confusion, le domaine des "études du milieu" en rendant obsolètes beaucoup d'analyses géographiques. "L'environnement bouscule les sciences", écrit J.C. Oppeneau, chef du SRETIE (Ministère de l'Environnement), et la géographie plus que d'autres.

Aujourd'hui, il nous reste à courir après le temps perdu. Certes, nous pouvons nous fonder sur les travaux avancés de géographes isolés, voire des laboratoires de géographie. Mais le temps des pionniers, les uns défrichant l'environnement forestier, les autres l'environnement urbain, d'autres encore les "espaces vécus" et les paysages, sans véritables connexions et sans aucune théorisation, est révolu. C'est l'ensemble de la discipline géographique qui doit, en tant que telle, sous son nom et en son nom, s'engager avec son corpus méthodologique et ses pratiques.

Dans ce foisonnement explosif et concurrentiel la géographie n'est pas spécialement attendue. Il n'y a pas, toute prête, de place à prendre. La géographie doit démontrer sa capacité à enrichir, ou à renouveler, les problèmes environnementaux. En répondant à plusieurs exigences.

\subsection{Conduire une démarche scientifique à trois niveaux}

- Au niveau épistémologique, participer plus directement et plus créativement au mouvement général des idées concernant les questions d'environnement, d'écologie, de qualité de la vie, etc. C'est un débat de société d'une ampleur planétaire qui met directement à contribution l'ensemble des connaissances et des pratiques géographiques. Il doit transiter par le filtre de l'épistémologie et de l'histoire des sciences, spécialités encore insuffisamment pratiquées en géographie.

- Au niveau disciplinaire, affirmer l'identité géographique en renonçant, une fois pour toutes, à embrasser la totalité de la problématique environnementale à toutes les échelles d'espace et de temps. Cela revient à dessiner un ou plusieurs itinéraires géographiques, scientifiquement balisés par des méthodes, des techniques et des pratiques, en distinguant bien ce qui relève de la recherche fondamentale, qui doit rester prioritaire dans le domaine de l'environnement, de la recherche finalisée, et de la participation directe à des expertises ou à des développements technologiques.

- Au niveau interdisciplinaire, participer à la réflexion et aux études au même titre que les autres sciences de la société et de la terre. La géographie doit d'abord tirer les conséquences du développement des autres disciplines, de l'extension de leurs concepts et de leurs champs d'investigation ainsi que des rapports qu'elles ont déjà noués entre elles. L'émergence de l'écologie scientifique doit être tout particulièrement prise en compte comme un facteur positif. De ce point de vue, le positionnement des géographes, chercheurs ou experts, dans les programmes interdisciplinaires nationaux (SRETIE, MRT, CNRS) ou internationaux (MAB, Global Change) doit être analysé avec beaucoup d'attention car il ne se fait pas souvent au bénéfice de la discipline et, 
de ce fait, n'est pas suffisamment reconnu à ce titre par la communauté scientifique et les grands organismes nationaux et internationaux qui ont l'environnement en charge.

\subsection{Mission pédagogique}

En plus de cette problématique d'amont, à caractère essentiellement scientifique, la géographie doit aussi remplir une autre mission, encore plus délicate, concernant la pédagogie. Dans ce domaine, la responsabilité assumée par la géographie est devenue écrasante et a menacé, à plusieurs reprises, de rompre l'équilibre de la discipline. Cette problématique d'aval, inhérente aux origines de la géographie, est liée au fait que les géographes sont chargés, à tous les niveaux d'enseignement, soit directement, soit indirectement par le biais de l'histoire, d'expliquer le monde aux jeunes générations. Les questions d'environnement y figurent très largement, bien que les géographes n'aient pas le monopole de cet enseignement. C'est ainsi que l'enseignant-géographe, qu'il soit bien ou mal formé, est quotidiennement amené à outrepasser les limites de la compétence scientifique de sa discipline (tectonique de plaques, "trou d'ozone", pollutions biochimiques, etc). Situation aberrante, intenable pour beaucoup de géographes et portant préjudice à la crédibilité de la discipline, qu'il faut dénoncer et corriger. D'une façon plus générale, la géographie a une fonction didactique qui relève de la culture générale et qu'il faut toujours avoir à l'esprit quand on traite de questions aussi médiatisées que celles de l'environnement.

Face à la poussée de l'environnement la géographie est, toutes choses égales, dans une situation analogue à celle qu'elle a connue, vers les années 60 , quand a émergé la notion d'aménagement du territoire. L'ambition avait été démesurée : faire de la géographie "la science de l'aménagement". Il a fallu en rabattre. Mais l'expérience a été salutaire et la géographie est aujourd'hui largement reconnue dans ce domaine.

\section{LA PERCÉE ENVIRONNEMEN. TALISTE : LES PRÉMICES D'UNE "RÉVOLUTION COPERNICIENNE"}

\subsection{L'environnement ambigu}

L'environnement, le terme et son usage, est une source intarissable de confusions, de digressions, de collusions. L'environnement est dans tout et réciproquement, au centre comme à la périphérie. C'est le type même du mot "compréhensif', c'est-à-dire de la notion-valise, débordant de sens et de contre-sens que tout le monde entend, mais que chacun comprend à sa guise. Son évolution sémantique est celle d'une dérive. Il a d'abord été l'environnement non-vivant (climat, roche, eau) des peuplements végétaux, puis des biocénoses, dans une conception étroitement naturaliste. Il est devenu ensuite, par extension, "l'environnement naturel", à forte connotation biologique, des hommes. Puis, il a pénétré le domaine social, économique et culturel, d'abord avec des implications biologiques, ensuite en investissant la "noosphère".

Cet expansionnisme, apparemment sans limites, est, sans aucun doute, un extraordinaire révélateur de "questions vives" et un moyen de remise en cause des comportements et des analyses scientifiques. Mais c'est aussi une emprise totalisante, toujours mal contrôlée, qui ferait lever de légitimes inquié- 
tudes chez les chercheurs en sciences humaines et sociales, et plus encore chez les philosophes, si, d'analogies en globalisations, la démarche environnementale n'en devenait souvent insignifiante, trop pleine de trop de vide et surtout, beaucoup plus dangereusement, trop moralisante et trop manichéenne. Il faut que les géographes surmontent leur réticence face aux technocraties et aux mystiques de l'environnement et contribuent à les exorciser. Car dédaigner ou minimiser l'environnement serait, pour la géographie, passer à côté d'une des grandes mutations, culturelle et scientifique, du monde contemporain.

\subsection{Une métaphysique planétaire}

Alors que de partout s'effondrent les idéologies familières, la montée en puissance de la notion d'environnement apparaît comme la grande révolution $\mathrm{du} \mathrm{XX}^{\mathrm{c}}$ siècle dans la façon de penser du monde et, plus particulièrement, les rapports de l'homme et de la nature. Tout se passe comme si la Terre, la Nature, les ciels et les oiseaux tournaient (on allait écrire... à nouveau) autour de l'Homme et dépendaient de plus en plus de lui, de son impact matériel comme de sa sensibilité et de ses phantasmes. D'objectif, le monde est devenu subjectif. Et quand toutes ces choses ne tournent plus tout à fait rond, on parle de crise ou de catastrophe d'autant que ces phénomènes se développent et interfèrent dans un monde que l'on sait fini, aux lendemains pour le moins incertains. Alors l'anthropocentrisme égoïste s'efface, sans contradiction apparente, devant un écocentrisme universaliste qui peut devenir tout aussi radical... et tout aussi ridicule. C'est ainsi que l'environnement, tel un concept mou, balance, sans principe, entre deux extrêmes.

Faut-il, pour autant, voir dans cette prise de conscience de la fragilité et de la finitude du monde la gestation d'une nouvelle idéologie? Pour le moins, la fin des idéologies de "progrès", positives et optimistes, marxistes ou libérales, qui ont accompagné et encensé la révolution industrielle. Et, dans le meilleur des cas, la naissance d'une éthique à base scientifique mais qui n'en reste pas moins, inévitablement, le vecteur d'idéologies incontrôlables et contradictoires, hyperprogressistes ou surtout ultraconservatrices à l'image des écologismes politiques tentés par tous les extrémismes. On ne doit pas, par scientisme ou par angélisme, considérer la recherche environnementale en dehors de son environnement éthique et métaphysique, comme si on pouvait facilement l'expurger de certains de ses élans mystiques. On ne fait pas de l'environnement sans conscience et le politique n'est jamais très loin.

\subsection{Une bifurcation épistémologique}

La pensée environnementale ne se réduit ni à un supplément d'âme ni à un supplément de science. C'est autre chose de bien plus important : une révolution dans la façon de concevoir et d'étudier le monde avec un statut général d'épistémologie des rapports entre l'homme social et la nature.

L'environnement marque, avant tout, le retour en force de la nature et $d u$ naturel que l'expansion économique apparemment illimitée avait effacés avec l'artificialisation croissante du monde. Les géographes sont directement et malheureusement concernés. 
C'est un cinglant démenti à une géographie à la Janus, où la géographie humaine et la géographie physique se sont délibérément tournées le dos et où l'élément naturel a été marginalisé et rendu socialement insignifiant.

L'environnement, du moins tel qu'il est conçu aujourd'hui, est devenu, après beaucoup de tergiversations, clairement anthropocentré et, dans une large mesure, sociocentré. La nature et les phénomènes naturels y sont, bien entendu, considérés en eux-mêmes et pour eux-mêmes, mais de plus en plus, dans une perspective sociale au sens large, à la fois économique et culturelle. Cela n'a pas été sans réticences de la part des scientifiques "durs", mais la "demande sociale", pour une fois, a été la plus forte et a même suscité la reconversion de nombreuses problématiques. La recherche en environnement est l'exemple même de la recherche interdisciplinaire affirmée puisqu'elle associe, en théorie du moins, les sciences sociales aux sciences de la nature.

Toutefois, "la recherche en environnement”, affirme J.C. Oppeneau, "n'est pas une discipline". Ce n'est pas une science, actuelle ou en devenir, ni même une métascience venant coiffer tout ou partie des sciences de la société et de la nature. Les dites "sciences de l'environnement" ne sont jamais que des regroupements interdisciplinaires de circonstance autour d'un programme de recherche, fondamental ou finalisé, d'une expertise ou d'une formation pédagogique (DEA, école doctorale). L'environnement ne correspond pas à un nouvel objet, complexe, qui s'ajouterait aux objets scientifiques plus "simples" déjà reconnus et aux sciences connexes.
L'environnement est, par delà l'interdisciplinarité, la prise en compte globale de phénomènes connus ou inconnus et l'étude privilégiée de leurs interactions. Dépassant le clivage des sciences positivistes, il retrouve l'unité du vivant et l'unité de la planète. Il n'y a pas d'environnement sans systémique. La notion d'environnement est essentiellement fonctionnelle et englobante... y compris le fonctionnement des "boîtes noires"!

En incitant les scientifiques à collaborer entre eux et les sciences de la nature à s'associer avec les sciences de la société, la recherche en environnement a franchi un seuil épistémologique majeur et a jeté les fondements d'une nouvelle façon de raisonner les sciences dans un contexte social donné. Le savoir scientifique y a acquis une nouvelle configuration et une nouvelle dimension. Certains philosophes y voient le passage d'un "contrat social" à un "contrat naturel". "L'histoire globale entre dans la nature, la nature globale entre dans l'histoire : voilà de l'inédit en philosophie" (M. Serres). Voilà aussi de l'inédit en géographie!

\section{UN OBJECTIF POUR LES GÉOGRAPHES : TERRITORIALISER L'ENVIRONNEMENT}

"La géographie, ça sert d'abord à parler du territoire" (Jean-Paul Ferrier)

Le dialogue entre l'environnement et la géographie auquel nous convie Géoforum 1991 s'éclaire d'un jour nouveau. D'une part, ce n'est pas un débat interdisciplinaire entre deux sciences qui s'affrontent ou collaborent au niveau des méthodes et des pratiques, c'est une confrontation dissymétrique entre, d'un côté, 
une discipline, la géographie, et de l'autre, un complexe épistémologique au plus haut niveau qui embrasse toute la réflexion et tous les développements scientifiques sur les rapports de l'homme et de son environnement. D'autre part, cet échange a toujours tendance à déborder le terrain scientifique et à se développer dans le domaine de l'éthique. Situation complexe, difficile à gérer, mais inévitable et enrichissante, dont il fallait préciser le statut pour éviter les atermoiements et les dérapages.

La géographie est aujourd'hui reconnue comme "la science sociale des territoires" (Colloque de Prospective, Ministère de la Recherche et de la Technologie, Paris, 1990). L'appréhension géographique de l'environnement doit se manifester essentiellement dans cette perspective. "Territorialiser" l'environnement, c'est à la fois l'enraciner dans la nature et dans la société en se donnant les moyens conceptuels et méthodologiques de faire avancer la connaissance environnementale dans ce domaine. Nous partirons de six concepts fondamentaux. Ils initient six filières autonomes mais solidaires autour du concept central de territoire.

\subsection{Socialiser l'environnement}

Cette démarche va dans le droit fil de l'évolution du "concept" d'environnement et elle ne relève pas de la seule géographie. Les anthropologues, les sociologues et les économistes ont déjà apporté leur contribution face à des naturalistes au début assez réticents. Socialiser l'environnement, c'est changer la finalité du système, dépasser la mésologie et l'éthnologie pour entrer dans l'analyse des stratégies sociales et des modes de représentation. Une grande partie de la géographie humaine, urbaine ou rurale, est directement mobilisable dans ce type de démarche au cours de laquelle elle rencontrera des conceptions de la nature plus assimilables pour elle que ne le sont beaucoup d'analyses de géographie physique.

\subsection{Spatialiser l'environnement}

La contribution de la géographie est ici essentielle, mais elle est loin d'avoir atteint son plein développement. On constate que de nombreuses études environnementales, soit flottent dans un espace mal déterminé, soit se limitent à un quadrillage de type chorologique hérité de la biogéographie. L'approche spatiale, qualitative ou quantitative, est devenue une connaissance au sens plein, riche d'implications et de prolongements sociaux et naturels dans laquelle beaucoup de géographes excellent.

\subsection{Anthropiser l'environnement}

Les environnements "naturels" au sens strict sont devenus l'exception. Les milieux dits "naturels", forêt, cours d'eau, littoraux, sont en fait largement et anciennement artificialisés. Leur structure même, et à plus forte raison leur fonctionnement, donc leur évolution, dépendent largement des conditions de leur aménagement et de leur gestion par les sociétés successives. Cet aspect essentiel de l'environnement n'est pas inconnu des écologues mais ils n'ont pas toujours les moyens conceptuels et méthodologiques d'en saisir la portée : soit ils la minimisent et se dispensent de l'étudier, soit ils en 
donnent des interprétations négatives et souvent catastrophistes.

\subsection{Hybrider l'environnement}

Chacun est aujourd'hui convaincu que l'environnement n'est ni naturel ni naturaliste, mais qu'il recèle toujours une part, plus ou moins grande, de naturel. C'est donc un produit d'interface qu'il faut traiter comme tel. Or, les concepts qu'on utilise pour l'appréhender relèvent généralement, soit des sciences de l'homme ou de la société, soit des sciences de la vie et de la terre. Le dialogue tourne souvent à la cacophonie. Il y a beaucoup de confusion dans le maniement des concepts voire dans le langage le plus commun. L'environnement doit soigner son vocabulaire. La géographie, plus que d'autres disciplines, a l'habitude de manier des concepts "mixtes" à la croisée de champs sémantiques et disciplinaires : cadre de vie, climax, contrainte, crise, milieu, paysage, potentialité, ressource, etc. Il est urgent que la recherche environnementale se prête à une clarification sémantique qui doit assurer, en priorité, la maîtrise de mécanismes conceptuels qui relèvent encore trop souvent de l'analogie et du réductionnisme.

\subsection{Historiser l'environnement}

C'est la suite logique des propositions précédentes. On ne peut socialiser et anthropiser l'environnement que si on l'inscrit dans la perspective du temps et de la durée. Ce temps est d'abord celui de la nature, périodisation de très longue durée et temps circulaire des saisons. Mais c'est surtout le temps de l'histoire des sociétés qui ont fait et défait leur propre environ- nement... ou celui des autres sociétés. C'est la mémoire des territoires et des terroirs, gravée dans l'espace comme dans l'esprit des hommes (Cl. et G. Bertrand - 1991).

Cette démarche relève autant des historiens, des archéologues, des préhistoriens, des palynologues, des climatologues que des géographes. Ces derniers ont prouvé très tôt leur capacité à traiter de ces questions qui réclament une double compétence, naturaliste et historique.

\subsection{Patrimonialiser l'environnement}

Les études environnementales sont conçues, directement ou indirectement, pour servir à l'action. Patrimonialiser, ce n'est donc pas seulement rechercher une explication dans le passé ou assurer une survivance, c'est projeter l'environnement dans un avenir qui ne peut être que d'évolution rapide et souvent de mutation. Cette dimension prospective, encore bien rare, est difficile à assurer avec suffisamment de sérieux scientifique, mais elle est indispensable. Sans elle, la recherche environnementale manquerait de sens et de sel.

\section{DIRECTIONS DE RECHERCHE POUR UNE PRATIQUE GÉOGRA. PHIQUE DE L'ENVIRONNEMENT}

Les quelques propositions qui suivent n'ont pas la prétention de couvrir la totalité du champ environnemental auquel pourrait prétendre la géographie si elle se donnait pour objectif central de "territorialiser" l'environnement. Il s'agit seulement de présenter un certain nombre de filières de recherche actuellement mises en œuvre, soit à titre personnel, soit plus 
généralement à titre collect if dans le cadre du CIMA-URA 366 CNRS. C'est une façon parmi d'autres d'aborder cette problématique complexe en s'attachant à proposer et à mettre en cuuvre un système paradigmatique cohérent et auto-suffisant.

\subsection{La maîtrise géographique de l'en- vironnement}

Elle passe par une reconstruction de la géographie physique et l'élaboration d'un corpus méthodologique identitaire.

Pour s'affirmer en tant que telle, la géographie doit apporter la preuve de sa spécificité et de son efficacité : c'est-àdire fonder son identité sur des résultats tangibles au travers d'une méthode originale. Depuis deux décennies, le CIMA-URA 366 a progressivement imaginé et mis en pratique un "paradigme d'interface" qui vient d'être présenté sous sa forme intégrale, quoique succinte, au Grand Colloque de Prospective que le Ministère de la Recherche et de la Technologie a consacré à la Géographie (Paris, 12 décembre 1990). Nous y renvoyons, en rappelant seulement trois postulats méthodologiques fondamentaux du point de vue des recherches sur l'environnement :

- c'est une méthode qui se situe dans la perspective de la géographie, "science sociale des territoires";

- c'est une méthode d'interface qui s'attache à approfondir l'analyse interactive des faits sociaux et des faits naturels; - c'est une méthode de complexité et de diversité qui essaie d'appréhender l'environnement spatial au travers d'un système conceptuel tridimensionnel : le géosystème, concept naturaliste ; le territoire, concept socio-économique ; le paysage, notion socio-culturelle.

Cette démarche revêt des aspects pratiques, scientifiques et didactiques, qui se développent à partir de différentes problématiques ou thématiques. Ne seront évoqués ici, à titre d'exemple, que quatre d'entre elles, particulièrement significatives du point de vue de l'environnement.

\subsection{L'aménagement du territoire et la qualité de la vie}

Il s'agit de retrouver la dialectique perdue entre l'environnement et l'aménagement. Entre l'environnement et l'aménagement existe une coupure conceptuelle et institutionnelle. Elle est en partie d'ordre historique. Le concept moderne d'aménagement est issu du grand mouvement volontariste qui a accompagné la reconstruction des économies après la seconde guerre mondiale et a impulsé les premières tentatives de planification des territoires. Les interrogations pratiques sur l'environnement et les premières politiques de "protection de la nature" sont, pour une large part, nées du dysfonctionnement même des aménagements, sous la forme marginale et floue des études dites d'"impact". Les véritables recherches environnementales n'ont débuté que beaucoup plus tard, vers les années 80 , le plus souvent indépendamment des recherches sur l'aménagement et à partir d'institutions politiques, administratives et scientifiques différentes.

Un rapprochement s'est esquissé ces dernières années, en particulier grâce au PIREN (CNRS) et au SRETIE (Ministère de l'Environnement) dans le cadre de programmes interdisciplinaires et le "Plan 
Vert" a pris en charge la dimension globale du problème. Toutefois, le mouvement est lent et encore incertain car il va à l'encontre des modes de pensée dominants, des structures en place et des intérêts immédiats des groupes de pression constitués autour des ministères, des comités scientifiques et des laboratoires.

Aujourd'hui encore, l'environnement et l'aménagement sont en grande partie séparés et considérés, au mieux, comme deux conceptions différentes, irréductibles pour certains, de l'analyse de l'espace : l'une serait positive, l'autre négative ; l'une économiciste, l'autre écologisante ; l'une progressiste, l'autre conservatrice. Ce jeu du ying et yang mériterait d'être poussé à son terme, c'est-à-dire jusqu'à retrouver l'unité fonctionnelle de l'espace. Mieux ciblée, la recherche y gagnera en efficacité sociale. "Aux déséquilibres mondiaux socio-économiques s'ajoutent donc, progressivement, des déséquilibres écologiques qui atteignent toutes les populations de la planète, mais encore plus gravement les peuples les plus démunis : déséquilibres socio-économiques et déséquilibres écologiques sont d'ailleurs étroitement dépendants et "l'inégalité écolo. gique est dramatique quand on regarde ce qui se passe dans les pays du Sud et dans les pays de l'Est" (A. Ruellan).

Les géographes sont particulièrement bien armés pour assurer la synergie environnement-aménagement autour du concept de territoire. Ce ne serait que retrouver le prémonitoire "mesnagement" d'Olivier de Serres et les applications que les forestiers français en ont donné pendant des siècles. Ainsi la géographie se positionnerait d'elle-même au centre du débat forte, d'une part, de sa double compétence sociale et naturaliste, et d'autre part, de sa maîtrise de l'espace au travers du concept de territoire et des technologies afférentes (cartographies et imageries satellitaires) (cf travaux des géographes de l'ORSTOM).

Le moment est donc venu de renverser la prublérnatique. Jusqu'à présent, la question environnementale n'était qu'un aspect subsidiaire de la politique d'aménagement, une sorte de correctif plus ou moins condescendant, la manifestation toujours un peu tardive d'un "supplément d'âme". Ce raisonnement qui a sous-tendu plus de deux décennies de recherches fondamentales et finalisées paraît s'inverser. C'est à partir d'une politique englobante d'environnement, fondée essentiellement sur la qualité de la vie, que l'on doit aujourd'hui aborder les questions d'aménagement du territoire. L'aménagement devient, en quelque sorte, une démarche conceptuelle, méthodologique et pratique, correspondant à la mise en œuvre d'une politique d'environnement. L'aménagement est alors conçu comme la science de gestion territoriale de l'environnement. Ce renversement de tendance est en cours en France, en particulier sous l'impulsion du SRETIE. Il n'est pas encore maîtrisé et doit être analysé et manié avec prudence. La géographie a beaucoup à gagner : une nouvelle base de réflexion épistémologique, des méthodes et des pratiques à renouveler et à adapter, des champs d'application pour ses technologies d'images. Un seuil est à franchir. Il implique, impérativement, de reconsidérer le rôle et le contenu de la géographie physique et d'ouvrir la géographie à deux domaines de recherche qu'elle maîtrise 
mal : le biologique et le sensible, sans lesquels on ne peut pas parler sérieusement de vie et de qualité de la vie.

\subsection{L'unité du territoire}

Elle consiste à surmonter la coupure entre l'urbain et le rural et redécouvrir l'environnement urbain.

Si la traditionnelle coupure entre géographie humaine et géographie physique tend, dans les meilleurs des cas, à s'estomper, la recherche géographique contemporaine fonctionne, en grande partie, sur la séparation entre des études à dominante urbaine et des études à dominante rurale. La nouvelle dialectique entre l'aménagement et l'environnement amène à s'interroger sur le bien fondé d'un découpage que la diminution de l'activité agricole et les nouvelles fonctions urbaines de l'espace rural ne justifient plus.

Si, dans l'ensemble, les études géographiques maîtrisent aussi bien l'aménagement du côté urbain que du côté rural, il n'en va pas de même pour l'environnement. L'environnement rural est surtout appréhendé, sans difficultés particulières mais sans grande efficacité au plan économique et social, à partir des données classiques de la géographie physique. On retrouve là les forêts, les sols, les eaux et les climats familiers au géographe et l'analyse paysagère permet d'aborder sans trop de peine le "vécu" des ruraux. Par contre, les questions biologiques, et pas seulement les pollutions, sont traitées avec beaucoup plus de difficulté. C'est un environnement où la vie et sa qualité ne sont pas parfaitement resituées.

L'environnement urbain est, quand à lui, peu étudié par les géographes. Les préoccupations et les méthodes de la géographie physique ont du mal à s'appliquer ici et les géographes laissent ainsi la ville ouverte à une "écologie urbaine" qui se cherche, non sans pertinence, mais non sans ambiguïté. Un nouveau comité scientifique du SRETIE se consacre à faire progresser la connaissance de l'environnement urbain. Les géographes y ont, a priori, une place qui reste à concrétiser par des études adaptées. Enfin, c'est en partant de la ville que l'on analysera avec le plus d'efficacité un espace rural qui perd de sa cohérence et qui, de plus en plus, n'existera en tant qu'environnement que par rapport à la réalité de l'urbain et à l'image de l'urbanité.

\subsection{L'anthropisation et la "mémoire des terroirs" : de l'espace-nature au territoire- artefact.}

L'impact des sociétés sur la nature est l'une des grandes "questions vives" du moment. Cette interrogation multiple a suscité de nombreuses et fructueuses études, depuis longtemps chez les préhistoriens, les archéologues et les géographes, plus récemment chez les historiens, les économistes et les juristes. Ces études sont aujourd'hui prolongées et confortées, avec des moyens sophistiqués et des ambitions globales, par des physiciens, des biologistes, des géologues, des climatologues, en particulicr dans lc cadre de programmes de recherche sur l'environnement planétaire ( $\mathrm{MAB}$, Global Change, etc).

Les géographes ont assez peu participé à ce mouvement comme s'ils n'avaient rien à prouver. Or, dans le domaine des relations des hommes avec leur milieu, leurs 
compétences sont anciennes et reconnues. Mais elles n'ont jamais été suffisamment rassemblées et théorisées pour constituer un corpus spécifique et innovant.

Par système anthropique, nous entendons l'ensemble des processus matériels et immatériels, d'origine sociale, économique ou culturelle, qui transforment l'espacenature en un environnement plus ou moins artificialisé. L'environnement se définit alors comme l'interface spatiale tapissée d'objets ou de phénomènes "mixtes" (S. Moscovici) qui relèvent à la fois de la nature et de la société, soit dans leur structure, soit dans leur fonctionnement.

La source, au sens propre comme au sens figuré, devient alors une ressource au sein d'un système social donné ou à l'interférence de plusieurs systèmes sociaux qui se la disputent. Le processus d'anthropisation peut être spécifié ; d'une part, en analysant les mécanismes de l'artificialisation; d'autre part, en resituant lartefact dans le ou les systèmes de valeurs correspondants. Cette recherche implique plusieurs lectures, simultanées et contradictoires d'une même "unité environnementale". Une sapinière est, en même temps, un écosystème, un paysage, une masse de production de matière ligneuse, un espace de chasse, etc, pour des acteurs différents, voire pour un seul et même acteur.

Cette méthode multidimensionnelle repose sur une base historique. Il faut faire parler la "mémoire des terroirs". Elle évite de traiter les problèmes d'environnement “à plat", sans profondeur historique, c'està-dire sans tenir compte des effets d'hystérésis et sans resituer les milieux dans une perspective évolutive. En particulier, comment pourrait-on parler de crise ou de catastrophe sans analyse préalable des durées et des rythmes dans le temps long (travaux de JP. Métailié, B. Desailly et S. Briffaud du CIMA) ?

\subsection{Un retour critique et constructif à la monographie géographique}

Il s'agit en fait de la recherche d'un modèle systémique d'analyse de l'environnement territorial.

Définir l'environnement territorial d'un groupe social consiste d'abord à réunir une information disparate provenant de sources et de disciplines diverses. Il reste à trouver un principe d'ordre, puis de généralisation, permettant d'appréhender la structure et le fonctionnement de ce système territorial. On peut faire appel à la chorologie, à la mésologie, à l'écosystémique, etc. Mais aucun de ces modèles ne rend compte de la totalité de l'environnement et en particulier de sa dimension socio-culturelle. L'ethnologie anthropologique fournit de bons exemples de monographies territoriales et les sociologues se sont aussi intéressés à ce problème ( $M$. Jollivet). Les géographes ont été des orfèvres en la matière. Entre 1890 et 1950 , une certaine conception de la monographie a fait l'efficacité et la gloire de la géographie régionale française. Mais, faute de renouvellement, elle a fait aussi sa ruine. Envisager de s'en inspirer relève aujourd'hui encore, pour beaucoup de géographes, de la provocation. Certes, les critiques étaient et restent fondées. Mais si on se donne la peine de les reformuler, on peut trouver, dans l'arsenal scientifique contemporain, des solutions innovantes. La monographie traditionnelle était discursive, descriptive, qualitative, analytique (les fameux 
"tiroirs" : relief, climat, population, économie) et insistait, au nom d'un "exceptionnalisme" mal formulé, sur l'unicité de la réalité observée. Il est aujourd'hui possible d'imaginer un modèle systémique qui évite la plupart de ces inconvénients. De plus, cette démarche nous conduit à réintroduire une part contrôlée de qualitatif et une certaine dose d'exceptionnalisme, par exemple dans l'appréhension des paysages (ex.: Gavarnie, grand site mondial).

Nous pouvons déjà construire des monographies environnementales finalisées qui, à différentes échelles de temps et d'espace, font progresser la connaissance fondamentale des territoires et peuvent servir d'aide à la décision (ex. : le Sidobre).

\section{CONCLUSION : L'ENVIRONNEMENT AU MITAN DU SOCIAL}

Longtemps naturel, l'environnement a envahi le social beaucoup plus que le social ne l'a investi. D'abord marge lointaine et facultative, devenue ensuite périphérie obligée et de plus en plus exigeante, l'environnement est aujourd'hui au cour du social, nœud gordien de la gestion du territoire et de la qualité de la vie.

Pour la géographie, se laisser porter par cette vague de fond ne suffit pas. Il lui faut s'investir dans l'environnement encore mieux qu'elle ne l'a fait pour l'aménagement. En affirmant son identité de "science sociale du territoire" et en la fondant sur une démarche spécifique qui lui permette de consolider sa participation à la recherche interdisciplinaire. Que cela ne soit pas encore, pour la discipline, une occasion perdue qui verrait l'environnement sortir irrémédiablement... du "territoire des géographes".

\section{RÉFÉRENCES}

F. Bel, J. Barruet, M.G. Durand, F. Véron : Modélisation d'un processus d'intégration de logiques antagonistes référant à l'interface économie-écologie, Congrès européen de Systémique, Lausanne 1989 . INRA - CEMAGREF, 12 pages

C. et G. Bertrand : La mémoire des terroirs in Pour une archéologie agraire, sous la direction de J. Guilaine 1991, Paris, Colin, pp. 11-17.

G. Bertrand : La nature en Géographie, un paradigme d'interface, 1991, Géodoc n 34, Institut de Géographie Université de Toulouse-Le Mirail, 16 pages. Grands colloques de Prospective du Ministère de la Recherche et de la Technologie : La Géographie, 12 décembre 1990.

CNRS - PIREN : Recherches sur l'environnement, Le courrier du CNRS, 1989, n 72, 119 pages.

CNRS - PIREN : Orientation et Programmation scientifique du Programme Environnement, Lettres et programmes interdisciplinaires de recherche du CNRS, Environnement n 1, 1991, CNRS, Paris, pp. 4-6.

J.M. Cusset : L'économie des ressources naturelles : éléments d'orientation bibliographique. Informations et commentaires, Ministère de l'Environnement, $\mathrm{n}^{\circ}$ 73,1990 .

F. Dagognet : Nature, 1991, Paris, Vrin, 234 p.

G. Guille-Escuret : Les sociétés et leurs natures, 1989, Paris, Colin, 182 p.

B. Kalaora : La nature des écosophies, 1991, REED. SRETI n ${ }^{\circ}$ 35, pp. 32-35.

B. Lalonde : Plan national pour l'environnement, 1990, Environnement-Actualité. nº spécial, $111 \mathrm{p}$.

J.C.Lévy : Le choix de la nature, 1991, REED. SRETIE n`35, pp. 30-32.

J.C. Oppeneau : L'environnement bouscule les sciences, 1991, Science et avenir, n 83 , pp. 96-98.

R.H. Peters : A critique for Ecology, 1991, Cambridge University Press, 440 p.

M.-C. Robic et al. : Les géographes français entre milieu et environnement, 1990, SRETIE, $276 \mathrm{p}$.

G. Rougerie, N. Beroutchachvili : Géosystèmes et paysages, 1991 ,Paris, A. Colin, 301 p.

A. Ruellan : Environnement et relations internationales, Letres et programme interdisciplinaires de recherche du CNRS Environnement, $n^{\circ} 2,1991$, CNRS, Paris, pp. 2-4.

M. Serres : Le contrat naturel, Paris, 1990, F. Bourin, $191 \mathrm{p}$. 